\title{
SPAGHETTI VS. MODULAR CODE - THE CHOICE OF NOVICE PROGRAMMERS
}

\author{
Ilana Lavy and Rami Rashkovits \\ Yezreel Valley College \\ Yezreel Valley, Israel
}

\begin{abstract}
Code modularity is one of the criteria for testing software quality. Nevertheless, novice programmers often tend to avoid writing modular code. In this study, we aim to explore the circumstances in which novice programmers choose to write modular code. To address this aim, two student groups, twenty in each, were given a programming task, each in different configuration. Group-1 was given the task in several phases, each add complexity to the previous one, while Group- 2 was given the entire task at once. The entire task was rather complex and its modular solution requires the definition of few classes and methods, each responsible to one of the problem's aspects. The students' solutions were analyzed using the dual-process theory, cognitive dissonance theory and content analysis methods to explore the extent of code modularity. The analysis revealed the following: (1) as regards to Group-1, a minor increase in the number of modular solutions was found while they progressed along the phases; (2) The number of modular solutions of Group-2 was higher than of Group-1. Analysis of students' justifications for lack of code modularity in Group-1 revealed the following. The first phase of the problem was perceived as rather simple hence many students did not find any reason to invest in designing a modular solution. When the task got complex in the following phases, some students realized that a modular solution would fit better, hence a cognitive dissonance was raised. Nevertheless, many of them preferred to continue their course of non-modular solution instead of re-designing a modular new one. In order to decrease the dissonance they claimed that this decision saved time. Students of both groups also attributed their non-modular code to lack of explicit criteria for the evaluation of the code quality that lead them to focus on functionality alone.
\end{abstract}

\section{KEYWORDS}

Code Modularity, Novice Programmer, Program Design, Cognitive Dissonance Theory, Dual-Process Theory

\section{INTRODUCTION}

Modular programming refers to a technique design that separates the functionality of a code into independent, extendible, reusable, maintainable and interchangeable modules, each responsible for one aspect of the desired behavior of the program (Parnas, 1972). Modular programming is a core issue in programming studies (Topi et al., 2010).

Code modularity supports gradual development and easy maintenance. In order to provide a modular code, one needs to possess abstract thinking capabilities enabling her to decompose the solution into small logical parts, and then integrate them to create the whole solution. The main challenge of this process is to define the 'right' and the 'right' functions with the 'right' parameters, allowing efficient integration. Modular design necessitates recursive decomposition of the problem into subcomponents, until the components are sufficiently simple and each responsible for a single operation.

Upon graduation, novice programmers are familiar with modularity principles and are aware of its importance to software quality; however, they often tend to avoid modular programming (Bollinger et al., 1999; Stamelos et al., 2002). Many students adapt 'bad programming habits' during their studies. These habits are the result of focusing on functionality only, while neglecting the structure and quality of the code. As far as they are concerned, if the program does what it should, its structure is not important, and hence it is not necessary to invest in design. 
In order to demonstrate their programming abilities novice programmers are focused mainly on being productive. Novice programmers assume that they are expected to provide the solution in the shortest possible time in order to prove their professionalism. To achieve this goal, they often skip the design phase that would bring them closer to a modular code. The result is what is called spaghetti code, with many repetitions of similar code snippets. We believe that in cases where novice programmers will recognize that they are facing a complex problem, such that modular design may shorten the coding time, they will choose to invest in it.

The aim of this study is to explore the conditions in which novice programmers choose to program modular or non-modular code and to understand the reasons underlying their choices.

To address this aim, two student groups, twenty in each, were given a programming task, which included repetitive components, each in different configuration. Group-1 was given the task in several stages, each add complexity to the previous one, while the Group-2 was given the entire task at once.

\section{BRIEF THEORETICAL BACKGROUND}

Modularity in software systems was widely discussed in the research literature and it was found that software systems with low degree of modularity have costly maintenance (Baldwin et al., 2000; Sullivan et al., 2001; MacCormack et al., 2007). On the other hand, researchers found that modular programming raises the quality of software packages by improving the software's readability and understanding, facilitates maintenance and increases the reuse of the software components (Sullivan et al., 2001; MacCormack et al., 2007).

In attempting to explain why novice programmers avoid modularity in spite their awareness to its importance we turned to the Dual-Process and Cognitive Dissonance theories. The Dual-Process theory deals with the distinction between intuitive and analytical modes of thinking. It suggests that human cognition and behavior operate in two different modes, in parallel, called System 1 and System 2 (Leron \& Hazzan, 2006; Kahneman, 2002). These modes are similar to our perception of intuitive and analytical modes of thinking (Fischbein, 1987); are activated by different parts of our brain, and have different evolutionary history. The Dual-Process theory refers to S1 thinking processes as being automatic and fast. They are perceived as unconscious, effortless, and inflexible. S2 thinking processes on the other hand, are being effortful and slow. In addition, they are perceived to be flexible, conscious, and expensive in terms of working memory resources. S1 and S2 systems differ mainly on the level of accessibility referring to the easiness and rapidness thoughts come to our mind.

While probing in the students' reflections expressed during the interviews, we noticed an underlying motive referring to a cognitive dissonance they experienced. Hence, we turned also to the cognitive dissonance theory to find answers. The cognitive dissonance theory refers to the mental discomfort that might occur to an individual who at the same time holds two or more incongruous beliefs, ideas, or values (Festinger, 1957; Cooper, 2011). Cognitive Dissonance theory refers to human tendency to strive for internal psychological consistency, in order to be able to function mentally. A person experiencing internal inconsistency becomes psychologically uncomfortable, and is tries to reduce the dissonance. This can be achieved by altering the stressful behavior, either by adding new information to the cognition causing the psychological dissonance to reduce the dissonance, and/or by actively avoiding contradictory information likely to increase the degree of the cognitive dissonance.

\section{THE STUDY}

\subsection{The Study Participants and Task}

Forty participants took part in the study, all were third (and final) year Information Systems students in an academic college, in the 2017-2018 years. 
The participants were randomly assigned into two groups (20 students in each). They were given a programming task, which was presented to each group in a different way. The students were asked to address the following problem: (1) input of a polynomial coefficients and a certain point $\mathrm{x}$; (2) compute the polynomial value at $\mathrm{x}$; (3) repeat the previous step for the first, second and third derivatives of the polynomial. Group-1 were provided with the task divided into three successive phases each was given after the completion of the previous. The first phase refers to steps 1-2. The second phase refers to step three with the first derivatives, and the last phase refers to the rest of the derivatives. Group- 2 had to address the same task, presented as a whole. Both groups were given no specific guidelines other than the requirement to provide a solution in any programming language they deem fit.

\subsection{Data Gathering and Analysis}

The research data included the students' solutions to the given tasks and the transcripts of the conducted interviews. Using content analysis methods (Neuendorf, 2002) the students' solutions were classified into three main categories: (1) non-modular; (2) partial-modular; and (3) full-modular. In the first category, we classified solutions in which the entire code was written in one or two functions. In the second category, we classified solutions in which several functions were defined, each responsible for small functionality, however, only one class exists representing both the program and the polynomial computations. In the third category, we classified modular solutions including at least two classes, one represents the program and the interaction with the user, and the second represents the polynomial. Moreover, each class includes several simple functions, each dealing with one functionality.

After classifying the students' solutions to the above categories, to understand the underlying reasons behind the students' choices of solution courses, we conducted open interviews with a representative group of students (three students from each category and each phase). The interviews transcripts were analyzed using te Dual-Process and the Cognitive Dissonance theories.

\section{RESULTS}

The results shown in Figure 1 demonstrate the evolution of modular solutions across the phases of Group- 1 . As shown, the number of non-modular solutions decreases from one phase to the next. However, it remains high even after the third phase in which the entire problem was revealed.

Figure 2 demonstrates the difference between the results of the final phase of Group- 1 in which the entire problem was revealed, and Group-2 that had to address the entire problem right from the beginning. Comparing group-1-phase 3 with Group-2 distributions reveals that although at this point both groups had the entire problem, there is a significant difference between the numbers of non-modular solutions between groups.

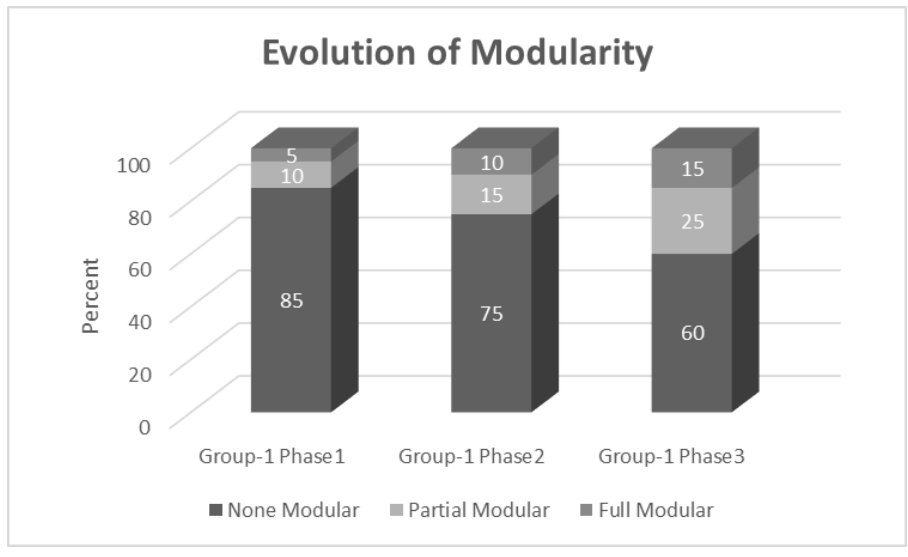

Figure 1. Evolution of Modularity across Phases (Group-1) 


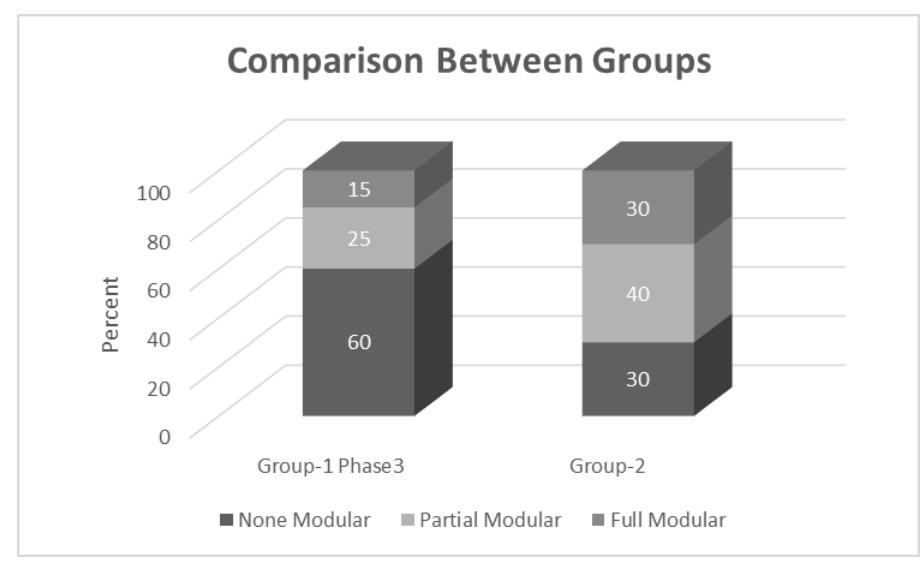

Figure 2. Comparing Group-1 and Group-2

\section{DISCUSSION}

As to phase-1 solutions, the high percentage of non-modular solution of phase-1 (Figure 1) may be attributed to the students' perception of the problem. The problem provided in the first phase was rather simple, without any repetitive structures. Hence, the students, being novice programmers, did not recognize the advantages of investing efforts in designing classes and functions that would not be used more than once. The following explanation was provided in similar words by these students: "Why not use a modular solution?.. I did not really think about it. This looks like a very simple task... If I knew there were follow-up tasks with repetitive structure, I might have done better." In terms of the dual-process theory (Gilovich, et al., 2002; Stanovich, \& West, 2000; Kahneman, 2002), we may say that the students who provided a non-modular solution acted in an intuitive manner (activating S1). On the other hand, those who have provided modular code introduced additional considerations related to the quality of the solution (activating S2), as some of these students uttered in similar words: "The task was very simple, so I figured out that you examine not only the solution but also its quality."

As to phase-2, some repetitive structures are revealed (i.e., the computation of the polynomial and its first derivative at point $\mathrm{x}$ ). The students were provided with their previous phase solutions after we took a snapshot of them. As can be seen from figure 1, non-modular solution are still in high percentage $(75 \%)$. In fact, they proceeded the first phase course of solution, adding the needed code to the main method, including code duplications. However, two students had improved their solution. One of them rewrote his solution and built a separate class to represent the polynomial function, and the other changed the code structure by separating the main method into several ones.

The students who provided non-modular solution to the phase-2 problem uttered in similar words: "In fact, it occurred to me that the code for the evaluation of the derivative polynomial is identical to part of the code I wrote earlier. However, since I already had a solution to the previous stage, it seemed a waste of time to rewrite the solution. Therefore, I decided to continue without changing the code from earlier stage." The above utterance imply on buds of understanding as regards to the need for modular solution. We may say that at this stage, a cognitive dissonance (Festinger, (1957; Cooper, 2011) regarding the effectiveness of their course of solution was emerged. To overcome this dissonance, they justified their adhering phase- 1 course of solution claiming that it would save time compared to the idea of starting the solution from the beginning. In terms of dual-process theory (Gilovich, et al., 2002; Stanovich, \& West, 2000; Kahneman, 2002), we may say that although they became aware to the need to change their course of solution, the temptation not to activate $\mathrm{S} 2$ was stronger since it saved investment of cognitive efforts. 
In phase-3 the students were asked also to calculate all the remaining derivative polynomials of the original polynomial and compute their values at the given point $\mathrm{x}$. The percentage of non-modular solutions was still high (60\%) (Figure 1). However, three additional students redesigned their solutions at this phase and provided modular (or partial-modular) solutions. Still, the tendency to invest minimal efforts in solving the task and avoid redesigning the entire solution was dominant.

During the interviews, one of the few students that changed their solution from non-modular to partial-modular uttered: "At this stage I noticed that repeated actions of polynomial derivation had to be done for the second time and I saw that the code I wrote for the previous stages began to be cumbersome with many repetitions. Therefore, I decided to write a function that calculates the derivative and avoid some of these repetitions. If I would get the entire problem from the start, I might design and implement the derivative method from scratch". As to these few students, we may say that to deal with the cognitive dissonance they experienced, they decided to abandon their intuitive thinking (activating S1) and adopt analytical thinking (activating S2).

As to the students of Group-1 who adhere their non-modular solution provided the following explanation in similar words: "At this stage it was obvious to me that the continuing with the same course of solution will lead to many code repetitions. However, I did not consider redesigning since I wanted to complete the task as fast as possible, having in mind that the most important goal is to solve the problem." At this phase, in which the entire problem was revealed to Group-1, most of the students who solved the previous phases in a non-modular way came to realization that their course of solution was problematic (code repetitions, unclear). We may say that they experienced a cognitive dissonance. To solve the dissonance, they decided to adhere the same course of non-modular solution (activating S1). They justified their choice claiming that redesigning the solution at this stage would take long time, more than to keep the same course.

Group-2 students were given the entire task, which was composed of all the three phases handed to Group-1. As can be seen in Figure 2, only $30 \%$ of the solutions were non-modular. The students, who provided a full-modular solution, uttered in similar words: "When I read the problem it occurred to me right away that many calculations of polynomials are required. Hence, I created a polynomial class to contain all the necessary methods, just as we learned in object-oriented programming course. It was not a difficult task for me."

As to the Group-2 students who provided non-modular solution, they justified their choice in similar words: "As I progressed with my solution, I saw that it contains many code repetitions. But you [the researchers] didn't specified any criteria as regards to the quality of the solution, so I decided to invest the minimal efforts to solve the problem... to provide a modular solution, it would have taken me longer time to achieve."

From the above utterances, we can conclude that though the students had to address the same problem, each of them interpreted it differently according to their capabilities and skills. Approximately Third of them were able to identify the problem components, define appropriate classes and methods and provide a full-modular solution. The first utterance can imply on the internalizing of the importance of early planning of the solution by third of the students, which eventually led to a full-modular solution. Yet, it was also important for these students to provide a solution in a short time. They understood that investing time in planning the solution would lead to saving in coding time and result in a short and clear solution. The internalization of the importance of the designing phase before coding can indicate on abstraction capabilities and planning skills. We may infer that the planning stage has become an integral part of the student's cognitive toolbox. In terms of dual-process theory (Gilovich, et al., 2002; Stanovich, \& West, 2000; Kahneman, 2002), we may say that for these students, full-modular solution is achieved by activating S1.

Figure 2 shows that more than a third of Group-2 students provided partial-modular solutions. They justified their course of solution uttering in similar words: "When I read the problem, I identified repeated elements such as the polynomial derivation and the calculation of its value. However, it was difficult for me to design a perfect solution that included many classes and methods. I did my best to finish the task successfully." We learn from the above utterance that these students understand the advantages of modular design but encounter difficulties in its implementation. In terms of the dual-process theory (Gilovich, et al., 2002; Stanovich, \& West, 2000; Kahneman, 2002), we might infer that modular design did not become completely an integral part of their intuitive thinking (S1).

As to the Group-2 students who provided a non-modular solution (30\%), we may infer that they were not able to grasp the problem as a whole, and instead they view it as a collection of sub-problems each to be addressed separately and successively, similar to the situation of group-1. In terms of dual-process theory 
(Gilovich, et al., 2002; Stanovich, \& West, 2000; Kahneman, 2002), we might infer that these students were not able yet to integrate modularity into their S1. This may be attributed to several reasons such as lack of solution planning before coding; not enough exposure to the consequences of non-modular code. As a result, the modularity principle was not completely internalized; and did not become an integral part of their cognitive toolbox.

\section{CONCLUSIONS}

In this study, we explored the circumstances in which novice programmers decide to design their course of solution before they start to code. The research data revealed that when a problem is simple, they tend to provide a quick solution, which lacks modularity. Even if they have to add to this solution a code that includes repetitive components, they adhere to the course of solution they had started with (Group-1 task). However, when the problem is handed to them as a whole (Group-2 task), approximately two-thirds of the students, invested efforts in planning and designing before coding their solutions and handed full or partial modular ones although it was not easy for them. To be able to design the solution before its implementation one has to possess abstraction and generalization skills. This is especially difficult when referring to novice programmers. The students behavior might be explained via dual-process theory (Gilovich, et al., 2002; Stanovich, \& West, 2000; Kahneman, 2002) referring to the natural tendency of people to invest minimal efforts while coping with cognitive tasks (activating S1). We also found that even after they understand that a modular solution would fit better, a situation, which evokes cognitive dissonance (Festinger, 1957; Cooper, 2011), they adhere the original course of the solution. To overcome the cognitive dissonance, many of them say that it seems ineffective and a waste of time to "throw away" what they have done so far and start all over again.

To raise students' awareness to the importance of code modularity and add this principle to their professional toolbox, we recommend the following action: (1) Include programming tasks that present two implementations of a given solution, one modular and the other non-modular. The students will be asked to put changes in the code, addressing new requirements. In such activities, students would appreciate the easiness of adding or changing functionalities to a modular code in contrast to the complexity of doing the same changes in the non-modular code; (2) Include tasks in which students will be required to reuse parts of code they provided in previous tasks. The aim of such activity is to raise the student's awareness to the crucial role of modularity in software reuse ; (3) Include tasks composed of several stages, in which each successive stage has to be handled by a different student. The student has to continue the solution of the next stage using the previous stage solution done by another classmate. The purpose of such activity it to confront students with the consequences of non-modular solutions.

In order to gain further refined insights, this study should be repeated with larger groups of students separating them according to academic achievements, and compare their performances to products of experienced programmers.

\section{REFERENCES}

Baldwin, C. Y. \& Clark, K. B. 2000. Design rules: The power of modularity (Vol. 1). Cambridge: The MIT Press.

Bollinger, T., Nelson, R., Turnbull, S. \& Self, K. 1999. From the editor-response: open-source methods: peering through the clutter. IEEE Software, vol. 16, no. 4, pp. 6-11.

Cooper, J. 2011. Cognitive dissonance theory. Handbook of theories of social psychology, vol. 1, pp. 377-398.

Festinger, L. 1957. A Theory of Cognitive Dissonance. California: Stanford University Press.

Fischbein, H. 1987. Intuition in science and mathematics: An educational approach (Vol. 5). Springer Science \& Business Media.

Gilovich, D. Griffin, \& D. Kahneman, (Eds.). 2002. Heuristics and biases: The psychology of intuitive judgment. Cambridge university press.

Kahneman, D. 2002. Maps of bounded rationality: A perspective on intuitive judgment and choice. Nobel Prize lecture, vol. 8, pp. 351-401. 
Leron, U. \& Hazzan, O. 2006. The rationality debate: Application of cognitive psychology to mathematics education. Educational Studies in Mathematics, vol. 62, no. 2, pp. 105-126.

MacCormack, A, Rusnak, J. \& Baldwin. 2007. The impact of component modularity on design evolution: Evidence from the software industry, Harvard Business School Working Paper.

Neuendorf, K.A. 2002. The Content Analysis Guidebook. Thousand Oaks, CA: Sage Publications.

Parnas, D.L. 1972. On the criteria to be used in decomposing systems into modules, Communications of the ACM, vol. 15, no. 9, pp. 1053-1058.

Stamelos, I., Angelis, L., Oikonomou, A. \& Bleris, G. L. 2002. Code quality analysis in open source software development". Information Systems Journal, vol. 12, no. 1, pp. 43-60, 2002.

Stanovich, E. \& West, R. 2000. Individual differences in reasoning: Implications for the rationality debate. Behavioral and brain science, vol. 23, pp. 645-726.

Sullivan, K.J., Griswold, W. G., Cai, Y. \& Hallen, B. 2001. The structure and value of modularity in software design, In the Proceedings of the $8^{\text {th }}$ European Software Engineering Conference, Vienna, Austria.

Topi, H., Valacich, J. S., Wright, R. T., Kaiser, K., Nunamaker Jr, J. F., Sipior, J. C., \& de Vreede, G. J. 2010. IS 2010: Curriculum guidelines for undergraduate degree programs in information systems. Communications of the Association for Information Systems, 26(1), 18. 